\title{
Reversing the Brazil-Nut Effect: Competition between Percolation and Condensation
}

\author{
A. P. J. Breu, H.-M. Ensner, C. A. Kruelle, and I. Rehberg \\ Experimentalphysik V, Universität Bayreuth, D-95440 Bayreuth, Germany
}

(Received 25 July 2002; published 10 January 2003)

\begin{abstract}
We report on experiments on vertically shaken binary granular mixtures, which separate into their components due to the external excitation. This well-known phenomenon, where large particles rise to the top of the mixture, is called the Brazil-nut effect. Recent theoretical findings predict also a reverse Brazil-nut effect, where large particles sink to the bottom of the container. We choose spherical beads of various diameters and materials in order to observe the transition from Brazil-nut effect to its reverse form. The direction of demixing depends sensitively on the external excitation, so that it is possible to switch between both effects for a given mass density ratio.
\end{abstract}

DOI: $10.1103 /$ PhysRevLett.90.014302

PACS numbers: 45.70.Mg, 05.65.+b, 45.70.Qj

Granular media consisting of small and large particles tend to demix when shaken vigorously enough. The Brazil-nut effect - if a particle mixture is shaken vertically, the larger particles will end up on top of the smaller ones, like the nuts in a muesli package - became the Drosophila melanogaster of granular media research [1-5]. Numerical simulations could validate the rise of larger particles [6,7]. Proposed mechanisms are, for example, convective motion of the smaller particles, which drag the larger ones to the top [3] or the filling of voids by the small particles, thereby lifting the larger ones [2,6,7]. Contrary to these common observations, Shinbrot [8], however, noticed that a large particle, depending on its density, could also sink to the bottom of the container. Recent theoretical investigations [9-11] explained that both effects, the rise or descent of the larger particles, may occur. The latter case has become known as reverse Brazil-nut effect. A simple relation between the size and density ratios of the particles predicts the borderline between both effects [9]. In this Letter we report on experimental tests of the validity - and limitations - of this theory.

One approach to describe an externally driven granular medium is to consider the individual particles as hard spheres. The driving could be - as in the case of the Brazil-nut effect - a vertical vibration of the container, which confines the granules. For strong driving, where all particles are in perpetual motion, the ideas of the kinetic theory of gases can be applied. Then it is possible to define a granular temperature $T$ in analogy to a gas, using the mean kinetic energy:

$$
T \propto \frac{1}{N} \sum_{i} \frac{m_{i}}{2}\left(v_{i}-\langle v\rangle\right)^{2}, \quad \text { with }\langle v\rangle=\frac{1}{N} \sum_{i} v_{i},
$$

where $N$ is the total number of particles, and $m_{i}$ and $v_{i}$ are the mass and the velocity of the $i$ th particle, respectively. Hong [12] calculated, on the basis of this model, a critical granular temperature $T_{c}=m g d \mu / \mu_{0}$, below which a system of monodisperse spheres condensate. This critical temperature depends on the diameter $d$ of the particles and the initial filling height $\mu$, measured in units of $d . g$ denotes the gravitational acceleration and $\mu_{0}$ is a constant, which depends on the spatial dimension and the underlying packing structure [12]. For a binary particle mixture different critical temperatures do exist, as pointed out by Hong et al. [9]. If a binary mixture of spheres is agitated by an external shaker, such that the granular temperature is in between the two critical values, one type of particle condenses while the other remains fluidized. Which particle species will condensate and therefore sink to the bottom of the container depends on the size and mass of the particles. Following Rosato et al. [6], Hong et al. [9] claimed that the crossover condition is given when the ratio of the critical temperatures is equal to the volume ratio of the two particle species, which leads to the simple relation

$$
\frac{d_{l}}{d_{s}} \approx\left(\frac{\rho_{l}}{\rho_{s}}\right)^{-1} \text {, }
$$

where $d_{l}, d_{s}, \rho_{l}$, and $\rho_{s}$ are the diameter and the mass density of the large $l$ and small $s$ particle, respectively. If the diameter ratio is smaller than the inverse of the density ratio the particle mixture should show Brazilnut effect and vice versa.

The prediction of formula (1) was put to the test by creating an instable layering and shaking of the container, as described in the following part. The experimental setup consists of a Perspex cylinder of $94 \mathrm{~mm}$ diameter and $200 \mathrm{~mm}$ height, which is mounted on an electromechanical vibration exciter (Brül \& Kjær, Type 4808). This container is shaken vertically by a sinusoidal modulation of the gravitational acceleration. The device operates at frequencies $f$ between 0 and $100 \mathrm{~Hz}$ and normalized accelerations $\Gamma=A(2 \pi f)^{2} / g$, where $A$ is the shaking amplitude and $g$ the gravitational acceleration. The acceleration $\Gamma$ is measured with an accelerometer attached to the base plate of the Perspex cylinder. The granular materials used were spherical particles with 
TABLE I. Spherical particles used in the experiment.

\begin{tabular}{lcc}
\hline \hline \multicolumn{1}{c}{ Material } & Density $\left(\mathrm{g} \mathrm{cm}^{-3}\right)$ & Diameter $(\mathrm{mm})$ \\
\hline Glass & 2.5 & $4,6,8,10,16,20,22$ \\
Aluminum & 2.6 & 2,6 \\
Bronze & 8.9 & 10 \\
Steel & 7.7 & $2,4,6,10$ \\
Polypropylene & 1.5 & $10,15,20$ \\
Polyurethane & 1.2 & 10,16 \\
Synthetic resin & 1.1 & 20 \\
Wood & 0.8 & 8,12 \\
\hline \hline
\end{tabular}

properties listed in Table I. In order to check whether a binary mixture would show Brazil-nut behavior or its reverse, we prepared a presumed instable configuration; i.e., we put several layers of the type, which were predicted by (1) to rise during shaking, on the bottom of the Perspex vessel. On top of these we placed one or more layers of the second type. If the prediction was correct, we would observe that the particles on top started to move through the layers of the other particle type, ending up at the bottom of the vessel (see Fig. 1). On the other hand, if the prediction turned out to be wrong the initial layering would be stable.

Most experiments have shown clearly either the Brazilnut effect [Fig. 1(a)] or the reverse form [Fig. 1(b)]. For some particle combinations a mixed state was stable. The results of the test and the theoretical borderline are shown in Fig. 2. For $82 \%$ of the tested combinations (145 out of 178) the prediction of (1) was correct. The prediction failed when one particle type was made of aluminum or polyurethane. We assume that for these materials the condition of hard spheres, which is one of the main propositions in the theory of Hong et al. [9] was not met. In the case of the reverse Brazil-nut effect it is crucial to choose an appropriate filling height. It turned

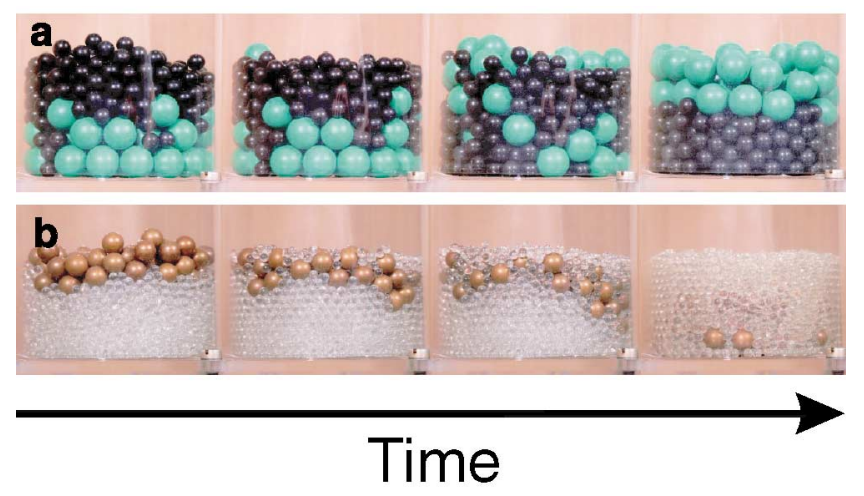

FIG. 1 (color). Temporal evolution of the system. (a) Initially $8 \mathrm{~mm}$ glass beads on top of $15 \mathrm{~mm}$ polypropylene, which show the classical Brazil-nut effect. (b) $10 \mathrm{~mm}$ bronze spheres on $4 \mathrm{~mm}$ glass beads showing the reverse Brazil-nut effect. out that the effect is completely destroyed, if the initially lower layer is too large.

The crossover condition was derived from the critical granular temperatures in the range $T_{\mathrm{c} 1}<T<T_{\mathrm{c} 2}$. In our experiments we deliberately left this regime by adjusting the amplitude and frequency. We prepared a random mixture of $10 \mathrm{~mm}$ polyurethane and $4 \mathrm{~mm}$ glass beads. The amplitude-frequency parameter space was explored by increasing the shaking amplitude quasistatically from zero to the maximal possible value, while keeping the frequency fixed. Four different configurations have been observed (Fig. 3): (i) At low amplitudes $(\Gamma<0.8 g)$ the particles stay at rest. (ii) Between $0.8 g$ and $1 g$ the larger particles start to wiggle around their initial positions. Because of this motion gaps are created, through which the small spheres can move downward and fill voids below. The downward motion occurs for a certain time, but ceases as the small particles reach again a stable configuration. We call this regime hindered Brazil-nut effect. (iii) Increasing the acceleration beyond $1 g$ results in rearrangements of the large spheres, ending in a hexagonal close-packed structure of the large spheres. In addition, large gaps are created for short times, such that the small particles are free to move downward. The final stable configuration is that of the Brazil-nut effect, where the small particles are at the bottom with the large ones on top. (iv) For accelerations larger than $3.5 \mathrm{~g}$ the smaller particles tend to move upwards. At sufficient high accelerations all small particles can pass the large ones. Once on top, they behave like a granular gas and the reverse Brazil-nut effect is established.

The transitions between the regimes (i), (ii), and (iii) do not depend on the driving frequency. The important

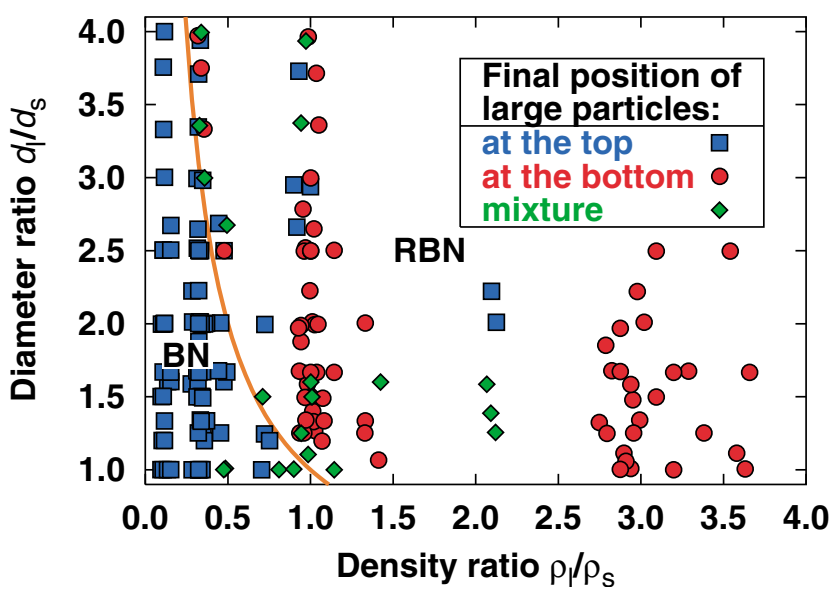

FIG. 2 (color). Phase space for particle properties. The plot shows the regimes where reverse (RBN) and classical Brazilnut effect $(\mathrm{BN})$ occurs depending on the particle properties. The solid line separating both areas is given by expression (1). Each symbol represents one experiment. Symbols on top of each other represent two different particle mixtures with the same diameter and density ratio, but show different behaviors. 


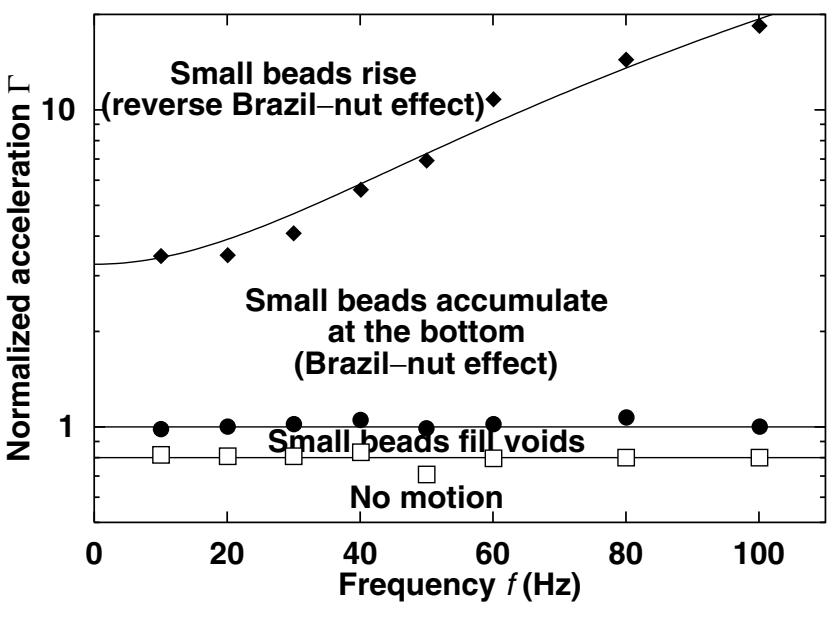

FIG. 3. Particle behavior for different driving modes of the shaker. The mixtures used are $10 \mathrm{~mm}$ polyurethane and $4 \mathrm{~mm}$ glass spheres. Lines are drawn to guide the eye.

control parameter is the acceleration. We found, however, that the transition between the Brazil-nut (iii) and its reverse effect (iv) depends on both acceleration and frequency.

Our experimental results confirm that the theory by Hong et al. [9] is able to predict the occurrence of the reverse Brazil-nut effect correctly. However, if the theoretical propositions (hard spheres, granular temperature $T$ between the critical temperatures) are not fulfilled, the entire scenario changes.

This work has been supported by the Deutsche Forschungsgemeinschaft RE-588/12.

Note added. - Recently we became aware of a controversy regarding the experimental verification of the reverse Brazil-nut effect [13-16]. We cannot explain why the experiment did not work in the case discussed there.

[1] J. C. Williams, Powder Technol. 15, 245-251 (1976).

[2] J. Duran, J. Rajchenbach, and E. Clément, Phys. Rev. Lett. 70, 2431-2434 (1993).

[3] J. B. Knight, H. M. Jaeger, and S. R. Nagel, Phys. Rev. Lett. 70, 3728-3731 (1993).

[4] M. E. Möbius, B. E. Lauderdale, S. R. Nagel, and H. M. Jaeger, Nature (London) 414, 270 (2001).

[5] M. Medved, H. M. Jaeger, and S. R. Nagel, Phys. Rev. E 63, 061302 (2001).

[6] A. Rosato, K. J. Strandburg, F. Prinz, and R. H. Swendsen, Phys. Rev. Lett. 58, 1038-1040 (1987).

[7] R. Jullien, P. Meakin, and A. Pavlovitch, Phys. Rev. Lett. 69, 640-643 (1992).

[8] T. Shinbrot and F. J. Muzzio, Phys. Rev. Lett. 81, 43654368 (1998).

[9] D. C. Hong, P.V. Quinn, and S. Luding, Phys. Rev. Lett. 86, 3423-3426 (2001).

[10] N. Shishodia and C. R. Wassgren, Phys. Rev. Lett. 87, 084302 (2001).

[11] J. A. Both and D. C. Hong, Phys. Rev. Lett. 88, 124301 (2002).

[12] D. C. Hong, Physica (Amsterdam) 271A, 192-199 (1999).

[13] G. A. Canul-Chay, P. A. Belmont, Y. Nahmad-Molinari, and J. C. Ruiz-Suárez, Phys. Rev. Lett. 89, 189601 (2002).

[14] P.V. Quinn, D. C. Hong, and S. Luding, Phys. Rev. Lett. 89, 189602 (2002).

[15] H. Walliser, Phys. Rev. Lett. 89, 189603 (2002).

[16] P.V. Quinn, D. C. Hong, and S. Luding, Phys. Rev. Lett. 89, 189604 (2002). 